\title{
A CRITERION FOR BEING A TEICHMÜLLER CURVE
}

\author{
Elise GOUjaRd
}

\begin{abstract}
Teichmüller curves play an important role in the study of dynamics in polygonal billiards. In this article, we provide a criterion similar to the original Möller's criterion, to detect whether a complex curve, embedded in the moduli space of Riemann surfaces and endowed with a line subbundle of the Hodge bundle, is a Teichmüller curve, and give a dynamical proof of this criterion.
\end{abstract}

\section{Introduction}

Given a curve in the moduli space of Riemann surfaces, we want to know whether it is a Teichmüller curve. By Deligne semisimplicity theorem the Hodge bundle over the curve decomposes into a direct sum of flat subbundles admitting variations of complex polarized Hodge structures of weight 1. Suppose that the restriction of the canonical pseudo-Hermitian form to one of the blocks of the decomposition has rank $(1, r-1)$. We establish an upper bound for the degree of the corresponding holomorphic line bundle in terms of the (orbifold) Euler characteristic of the curve. Our criterion claims that if the upper bound is attained, the curve is a Teichmüller curve.

For those Teichmüller curves which correspond to strata of Abelian differentials our criterion is necessary and sufficient in the sense that if the curve is a Teichmüller curve, then the decomposition of the Hodge bundle necessarily contains a non-trivial block of rank $(1,1)$ corresponding to the tautological line bundle for which the upper bound is attained.

The original criterion in the same spirit was found by Martin Möller in [10, Th. 2.13 and 5.3] where the condition detecting a Teichmüller curve is formulated in terms of Higgs bundle, or equivalently in terms of non-vanishing of the second fundamental form (Kodaira-Spencer map). In [17], Wright gives an alternative version of Möller's criterion, in terms of non-vanishing of the period map.

The key idea of our criterion is based on Forni's observation that the tautological bundle on a Teichmüller curve is spanned by those vectors of the Hodge bundle which have the maximal variation of the Hodge norm along the Teichmüller flow.

We combine this result of Forni with the Bouw-Möller version of the Kontsevich formula for the sum of the Lyapunov exponents of the Hodge bundle along the Teichmüller geodesic flow. Similar to the criteria mentioned above, the fact that the Teichmüller metric coincides with the Kobayashi metric will be crucial for the proof.

Received by the editors June 4, 2012. 


\section{Criterion}

Having a Riemann surface $X$, the natural pseudo-Hermitian intersection form on $H^{1}(X, \mathbb{C})$, is defined on closed 1 -forms representing cohomology classes as:

$$
\left(\omega_{1}, \omega_{2}\right)=\frac{\mathrm{i}}{2} \int_{X} \omega_{1} \wedge \overline{\omega_{2}}
$$

Restricted to $H^{1,0}(X, \mathbb{C})$, the form is positive-definite, and restricted to $H^{0,1}(X, \mathbb{C})$, the form is negative-definite.

This pseudo-Hermitian form of signature $(g, g)$ induces a form on the Hodge bundle $H^{1}$ over the the moduli space $\mathcal{M}_{g}$ of Riemann surfaces of genus $g$, where the fiber $H_{X}^{1}$ of the Hodge bundle over a point $X$ in $\mathcal{M}_{g}$ is $H^{1}(X, \mathbb{C})$. The pseudo-Hermitian form is covariantly constant with respect to the Gauss-Manin flat connexion on the Hodge bundle.

Let $\mathcal{C}$ be a complex curve in $\mathcal{M}_{g}$. We want to detect, whether $\mathcal{C}$ is a Teichmüller curve or not. Throughout this paper we assume that the genus $g$ is strictly greater than 1 since in genus one the problem becomes trivial: the moduli space $\mathcal{M}_{1,1}$ is a complex curve itself.

By Deligne semisimplicity theorem [2, Prop. 1.13], the Hodge bundle over $\mathcal{C}$ splits into a direct sum of orthogonal flat subbundles, such that the restriction of the canonical pseudo-Hermitian form to each subbundle is non-degenerate. Assume that this splitting contains a flat subbundle $\mathbb{L}$ of rank $r$, where $r \geq 2$, such that the signature of the canonical pseudo-Hermitian form restricted to $\mathbb{L}$ is $(1, r-1)$. Let us define $\mathbb{L}^{1,0}=\mathbb{L} \cap H^{1,0}$ and $\mathbb{L}^{0,1}=\mathbb{L} \cap H^{0,1}$. Deligne semisimplicity theorem combined with our assumption on the signature implies that $\mathbb{L}^{1,0}$ is a holomorphic line bundle over $\mathcal{C}$.

Note that for Teichmüller curves corresponding to the strata of Abelian differentials the splitting is always non-trivial, since it contains a flat subbundle of rank 2 such that the restriction of the pseudo-Hermitian form to this subbundle has signature $(1,1)$. The corresponding line bundle $\mathbb{L}^{1,0}$ is the tautological line bundle over the Teichmüller curve.

The curve $\mathcal{C}$ may have a finite number of cusps and conical points, so we need to consider the Deligne extension of the holomorphic line bundle $\mathbb{L}^{1,0}$, denoted by $\overline{\mathbb{L}^{1,0}}$ : it becomes an orbifold vector bundle at the cusps and conical points. So it has an orbifold degree, which in general is not an integer, but a rational number.

Let $\chi(\mathcal{C})$ be the generalized Euler characteristic of $\mathcal{C}$ : it is given by the formula

$$
\chi(\mathcal{C})=2-2 g-n_{\mathcal{C}}+\sum_{i}\left(k_{i}-1\right)
$$

where $n_{\mathcal{C}}$ is the number of cusps on $\mathcal{C}$, and $2 \pi k_{i}$ is the cone angle of the $i$ th conical point.

Theorem. If $\chi(\mathcal{C}) \geq 0$, then $\mathcal{C}$ is not a Teichmüller curve.

Suppose that $\chi(\mathcal{C})<0$. For any flat subbundle $\mathbb{L}$ of the Hodge bundle over $\mathcal{C}$ satisfying the above assumptions, one has

$$
\operatorname{deg} \overline{\mathbb{L}^{1,0}} \leq-\frac{\chi(\mathcal{C})}{2}
$$

If the equality is attained, then $\mathcal{C}$ is a Teichmüller curve, and the line bundle $\mathbb{L}^{1,0}$ is the tautological bundle. 
Any Teichmüller curve corresponding to a stratum of Abelian differentials admits a flat subbundle $\mathbb{L}$ of the Hodge bundle satisfying the above conditions, such that

$$
\operatorname{deg} \overline{\mathbb{L}^{1,0}}=-\frac{\chi(\mathcal{C})}{2}
$$

The first statement of the theorem results from the fact that any Teichmüller curve has negative curvature, or equivalently, an orbifold genus strictly greater than 1 . So from now on, we assume that $\chi(\mathcal{C})<0$, that is, $\mathcal{C}$ is hyperbolic.

Remark 1. We have to admit that our criterion does not directly detect Teichmüller curves corresponding to the strata of quadratic differentials. However, the canonical double covering construction associates to every such Teichmüller curve $\mathcal{C}$ in $\mathcal{M}_{g}$ a Teichmüller curve $\mathcal{C}^{\prime}$ in $\mathcal{M}_{g^{\prime}}$ in the moduli space of curves of larger genus, such that the new Teichmüller curve already corresponds to some stratum of Abelian differentials, and thus, would be detected by our criterion. The new Teichmüller curve $\mathcal{C}^{\prime}$ is isomorphic to the initial curve $\mathcal{C}$.

Since any Riemann surface $X^{\prime}$ in the family $\mathcal{C}^{\prime}$ admits a holomorphic involution which changes the sign of the corresponding Abelian differential, the Teichmüller curves corresponding to such a double covering construction can be identified. Thus, indirectly the criterion detects all Teichmüller curves.

Remark 2. As Martin Möller pointed out to the author, in the case $r=2$ this theorem can be refound by algebraic methods. Inequality (2.1) is a specific case of Arakelov inequality (see e.g., [2, Lemme 3.2]), and the bound is attained if and only if $\mathbb{L}$ is maximal Higgs (see $[12,15]$ for generalization in higher dimension). So Möller's criterion applies here and gives the conclusion of the theorem.

\section{Comparison of hyperbolic versus Teichmüller metric}

Recall that at any point $X \in \mathcal{M}_{g}$ the tangent space $T_{X} \mathcal{M}_{g}$ is identified with the space of essentially bounded Beltrami differentials, which is in duality with the space of integrable quadratic differentials on $X$ by the pairing $\langle\mu, q\rangle=\int_{X} q \mu$. So the cotangent bundle $T^{*} \mathcal{C}$ of $\mathcal{C}$ can be viewed as a suborbifold of $\mathcal{Q}_{g}$, the moduli space of quadratic differentials. We will denote the total space of the cotangent bundle to the curve $\mathcal{C}$ by $\tilde{\mathcal{C}}$, and points of $\tilde{\mathcal{C}}$ by $(X, q)$, where $X$ is a Riemann surface and $q$ is a quadratic differential on $X$. The pullback of $\mathbb{L}$ to $\tilde{\mathcal{C}}$ will also be denoted by $\mathbb{L}$. In the following, we will identify the cotangent bundle $\tilde{\mathcal{C}}$ with the tangent bundle by duality, so a quadratic differential will be seen as a tangent vector to $\mathcal{C}$.

We start by comparison of the two natural metrics on $\mathcal{C}$ : the canonical hyperbolic metric given by Riemann's uniformization theorem, and the induced Teichmüller metric. Both of these metrics are, infinitesimally, Finsler metrics, so they define norms on each tangent space $T_{X} \mathcal{C}$ of $\mathcal{C}$.

Lemma 1. Globally, on $\mathcal{C}$, the hyperbolic metric is larger than the induced Teichmüller metric, that is, the hyperbolic distance between any two points is larger than the Teichmüller distance.

Infinitesimally, on each tangent space $T_{X} \mathcal{C}$, the unit ball for the norm associated to the hyperbolic metric is included in the unit ball for the norm associated to the induced Teichmüller metric. 
Proof. The proof is based on the notion of Kobayashi metric (cf [7]). The canonical hyperbolic metric on $\mathcal{C}$ is by definition the Kobayashi metric on $\mathcal{C}$, and by Royden's theorem, the Teichmüller metric is the Kobayashi metric on $\mathcal{M}_{g}$. So the statement of the lemma results from the property of contraction of the (global or infinitesimal) Kobayashi metric for the inclusion $\mathcal{C} \hookrightarrow \mathcal{M}_{g}$.

Now we apply this lemma to the cotangent bundle $\tilde{\mathcal{C}}$, using the identification $\tilde{\mathcal{C}} \simeq T \mathcal{C}$.

Corollary 1. Let $\gamma(t)$ be a geodesic on $\mathcal{C}$ for the hyperbolic metric. Let us denote by $\gamma(\tau)$ the same curve parameterized by the arc length for the Teichmüller metric restricted to $\mathcal{C}$. The corresponding derivatives will be denoted by $\gamma^{\prime}=\frac{\partial \gamma}{\partial t}$ and $\dot{\gamma}=\frac{\partial \gamma}{\partial \tau}$. Let $v$ be an element of $\mathbb{L}$ at $\left(\gamma(0), \gamma^{\prime}(0)\right)=\left(X, q /\|q\|_{\text {hyp }}\right) \in \tilde{\mathcal{C}} \subset \mathcal{Q}_{g}$. Then the Lie derivatives of the norm of $v$ along $\gamma$ satisfy

$$
\left|\mathcal{L}_{\gamma^{\prime}(0)} \log \|v\|\right| \leq\left|\mathcal{L}_{\dot{\gamma}(0)} \log \|v\|\right| \text {. }
$$

Proof. Note that $\gamma^{\prime}(0)$ and $\dot{\gamma}(0)$ are tangent vectors to the same curve parametrized in two ways, at the same point, so they are colinear:

$$
\gamma^{\prime}(0)=\alpha \dot{\gamma}(0)
$$

Since $\gamma^{\prime}(0)$ is unitary for the hyperbolic metric, and $\dot{\gamma}(0)$ unitary for the Teichmüller metric, by Lemma $1,|\alpha| \leq 1$. The conclusion follows by the chain rule.

Note that Corollary 1 is valid for any choice of the norm in the Hodge bundle provided the norm varies smoothly with respect to a variation of a point in the base of the bundle. In the next section we pass to a very special Hodge norm.

\section{Variation of the Hodge norm}

The natural Hermitian form is positive-definite on $H^{1,0}(X, \mathbb{C})$, so it induces a norm:

$$
\|h\|_{1,0}^{2}=\frac{\mathrm{i}}{2} \int_{X} h \wedge \bar{h}
$$

Similarly, the intersection form is negative-definite on $H^{0,1}$ so its opposite defines a norm $\|\cdot\|_{0,1}$ on $H^{0,1}$. Note that for every $h \in \mathbb{L}^{1,0}$, we have $\|\bar{h}\|_{0,1}=\|h\|_{1,0}$. We define the Hodge norm on $H^{1}(X, \mathbb{C})$ by $\|v\|=\|h\|_{1,0}+\|a\|_{0,1}$, where $h$ is the holomorphic part of $v$ and $a$ the anti-holomorphic part. This is the norm that we will consider on $\mathbb{L}=\mathbb{L}^{1,0} \oplus \mathbb{L}^{0,1}$, by restriction. From now on we consider only the Hodge norm.

The second lemma gives a uniform bound for the variation of the Hodge norm in the direction of the Teichmüller flow (for the definition of the Teichmüller flow, see e.g., [4, Section 1]).

Lemma 2 (G. Forni). Let $v$ be a non-trivial element of the fiber $H^{1}(X, \mathbb{C})$ at $(X, q) \in \tilde{\mathcal{C}}$. Then the Lie derivative of the Hodge norm of $v$ along the Teichmüller flow satisfies the following inequality:

$$
|\mathcal{L} \log \|v\|| \leq 1
$$

Moreover, it is an equality if and only if $q=\omega^{2}$ with $\omega \in H^{1,0}(\mathbb{C})$ and

$$
v \in \operatorname{Span}_{\mathbb{C}}(\omega) \oplus \operatorname{Span}_{\mathbb{C}}(\bar{\omega})-\{0\},
$$

where $\operatorname{Span}_{\mathbb{C}}(\omega)$ is the tautological bundle. 
The statement is the extension to the complex case of a lemma of G. Forni (see [4, Lemma 2.1']), reformulated in [5, Cor. 2.1]. The original statement holds in $H^{1}(\mathbb{R})$ (with the Hodge norm), and by the Hodge representation theorem, it also holds in $H^{1,0}$, endowed with the norm $\|\cdot\|_{1,0}$. By conjugation, we obtain the result in $H^{0,1}$ with the norm $\|\cdot\|_{0,1}$. Finally, note that inequality (4.1) is equivalent to the following:

$$
|\mathcal{L}\|v\|| \leq\|v\|
$$

So, applying this majoration to each component (holomorphic and antiholomorphic) of an element $v$ of $H^{1}(\mathbb{C})$, endowed with the chosen norm $\|$.$\| , we obtain the following$ inequalities:

$$
|\mathcal{L}\|v\||=\left|\mathcal{L}\|h\|_{1,0}+\mathcal{L}\|a\|_{0,1}\right| \leq\left|\mathcal{L}\|h\|_{1,0}\right|+\left|\mathcal{L}\|a\|_{0,1}\right| \leq\|h\|_{1,0}+\|a\|_{0,1}=\|v\|,
$$

so the result holds in $H^{1}(\mathbb{C})$.

Note that there is another proof of inequality (4.1) in [9, Lemma 6.10], in terms of curvature of the metrics.

With this two lemmas we can achieve the proof of the theorem.

\section{Criterion in terms of Lyapunov exponents}

In this section we give an alternative version of the criterion, in terms of Lyapunov exponents. This version does not require any assumption on the signature of the pseudo-Hermitian form on the flat bundle $\mathbb{L}$, so it is more general, but it has less interest in practice, because Lyapunov exponents are harder to compute than orbifold degree.

Proposition. Let $\mathcal{C}$ be a curve in the moduli space $\mathcal{M}_{g}$, with $\chi(\mathcal{C})<0$, endowed with a flat subbundle $\mathbb{L}$ of rank $r \geq 2$ of the complex Hodge bundle, equivariant for the Gauss-Manin connection. Consider the Lyapunov exponents associated to the parallel transport of fibers of $\mathbb{L}$ along the geodesic flow given by the hyperbolic metric on $\mathcal{C}$. The absolute values of all these Lyapunov exponents are bounded above by 1 . If the bound is achieved, the curve $\mathcal{C}$ is a Teichmüller curve.

Proof. Let us first explain where these Lyapunov exponents come from. Recall that $\mathcal{C}$ is endowed with a canonical hyperbolic metric, which gives us a geodesic flow $g_{t}^{\text {hyp }}$ on $T_{1} \mathcal{C}$, the unit tangent bundle of $\mathcal{C}$, and by duality, on the unit cotangent bundle $\tilde{\mathcal{C}}^{(1)}$. We look at the parallel transport of fibers of $\mathbb{L}$, endowed with the Gauss-Manin connection, along this geodesic flow.

Let $\nu$ be the Liouville measure on $\tilde{\mathcal{C}}^{(1)}$.

Since $\mathbb{L}$ inherits a variation of the Hodge structure from the Hodge bundle, it has quasi-unipotent monodromy around any cusp (see [14, Th. 6.1]). So there exists a finite unramified cover $\hat{\mathcal{C}}$ of $\mathcal{C}$, such that the pullback of $\mathbb{L}$ on $\hat{\mathcal{C}}$ has unipotent monodromy around any cusp of $\hat{\mathcal{C}}$. Passing to this finite cover preserves the ergodicity of the geodesic flow that we consider on $\hat{\mathcal{C}}$ (because of the hyperbolic features of the geodesic flow and Hopf's argument, see e.g., Wilkinson's article [16]), and does not change the Lyapunov exponents. Actually, all results we will obtain on $\mathcal{C}$ lift to $\hat{\mathcal{C}}$, so up to passing to this cover, we will assume in the rest of this paper that the monodromy of $\mathbb{L}$ is unipotent around any cusp of $\mathcal{C}$.

So with this assumption and thanks to the majoration given by Corollary 1 and Lemma 2, the cocycle associated to the geodesic flow is log-integrable. The Oseledets 
theorem (see [11]) can be applied to this complex cocycle. We denote by $\lambda_{i}$ the Lyapunov exponents and $E_{\lambda_{i}}$ the corresponding subspaces.

By definition every vector $v$ in $E_{\lambda_{i}}(q)$ expands with the rate:

$$
\lambda_{i}=\lim _{t \rightarrow \infty} \frac{1}{t} \log \left\|v\left(\gamma_{q}(t)\right)\right\|
$$

where $\gamma_{q}$ is the geodesic for the hyperbolic metric starting at point $X$ in the direction $q$.

We can write:

$$
\lambda_{i}=\lim _{T \rightarrow \infty} \frac{1}{T} \int_{0}^{T} \frac{d}{d t} \log \left\|v\left(\gamma_{q}(t)\right)\right\| d t .
$$

So we have the following majoration:

$$
\lambda_{i} \leq \lim _{T \rightarrow \infty} \frac{1}{T} \int_{0}^{T} \max _{v \in \mathbb{L}}\left(\frac{d}{d t} \log \left\|v\left(\gamma_{q}(t)\right)\right\|\right) d t
$$

By Birkhoff's theorem, we have:

$$
\lim _{T \rightarrow \infty} \frac{1}{T} \int_{0}^{T} \max _{v \in \mathbb{L}}\left(\frac{d}{d t} \log \left\|v\left(\gamma_{q}(t)\right)\right\|\right) d t=\int_{\tilde{\mathcal{C}}^{(1)}} \max _{v \in \mathbb{L}}\left(\mathcal{L}_{\gamma_{q}^{\prime}(0)} \log \|v(q)\|\right) d \nu(q) .
$$

It results from Corollary 1 and Lemma 2 that

$$
\max _{v \in \mathbb{L}}\left|\mathcal{L}_{\gamma_{q}^{\prime}(0)} \log \|v(q)\|\right| \leq 1
$$

so

$$
\int_{\tilde{\mathcal{C}}^{(1)}} \max _{v \in \mathbb{L}}\left(\mathcal{L}_{\gamma_{q}^{\prime}(0)} \log \|v(q)\|\right) d \nu(q) \leq 1
$$

Similary, we have:

$$
\lambda_{i} \geq \int_{\tilde{\mathcal{C}}^{(1)}} \min _{v \in \mathbb{L}}\left(\mathcal{L}_{\gamma_{q}^{\prime}(0)} \log \|v(q)\|\right) d \nu(q) \geq-1 .
$$

So we obtain that $\left|\lambda_{i}\right|$ is bounded by 1 . Assume now that the bound is achieved for some index $i$. By symmetry of the spectrum (see [6, Th. 4]), the exponent $-\lambda_{i}$ lies in the spectrum, so we can assume that $\lambda_{i}=1$. It means that inequalities (5.2) and (5.5) are in fact equalities. Since the measure $\nu$ is normalized, and the modulus of the integrand in (5.5) is 1 at most (cf. (5.4)), it is almost everywhere equal to 1 and hence, by continuity, everywhere equal to 1 . It means that inequalities of Corollary 1 and Lemma 2 are equalities.

The first equality case in Corollary 1 implies that the two metrics (hyperbolic and Teichmüller) coincide on $\mathcal{C}$. Hence $\mathcal{C}$ is invariant by the Teichmüller flow, so it is a Teichmüller curve.

Let us denote by $v_{1}$ the element of $\mathbb{L}$ at point $(X, q)$ which maximizes the quantity $\mathcal{L} \log \|v\| \in[-1,1]$. Similarly, considering inequation (5.6) with $\lambda_{i}=-1$ gives an element $v_{2}$ which minimizes the same quantity. Clearly, $v_{1}$ and $v_{2}$ are independent. By Lemma 2, we have $q=\omega^{2}$ and $\operatorname{Span}_{\mathbb{C}}\left(v_{1}, v_{2}\right)=\operatorname{Span}_{\mathbb{C}}(\omega, \bar{\omega})$. So we obtain the additional information that $\mathbb{L}$ contains the complex tautological bundle. In particular, $\mathbb{L}^{1,0}$ corresponds to $\operatorname{Span}_{\mathbb{C}}(\omega)$. 


\section{End of the proof}

We will finish the proof of the theorem using that, with the additional assumption on the signature, there is only one non-negative Lyapunov exponent, which can be written in terms of degree of the line bundle $\mathbb{L}^{1,0}$. So we will be able to conclude with the previous proposition.

Since the pseudo-Hermitian form has signature $(1, r-1)$ on $\mathbb{L}$, there is at most one positive Lyapunov exponent, denoted by $\lambda_{1}$ (see [6, Th. 4]).

As the monodromy is unipotent around cusps, the degree of the extended line bundle $\overline{\mathbb{L}^{1,0}}$ is the integral on $\mathcal{C}$ of the curvature form $\alpha$ (first Chern class), cf [13, Prop. 3.4]. Then one has:

$$
\int_{\mathcal{C}} \alpha=\operatorname{deg} \overline{\mathbb{L}^{1,0}}
$$

We use now the formula for the sum of the Lyapunov exponents of an invariant subbundle with respect to a geodesic flow defined by the hyperbolic metric on the curve $\mathcal{C}$. This formula was outlined by Kontsevich in [8], developed by Forni in [4]. Bouw and Möller suggested in [1] an algebro-geometric interpretation of the numerator as the orbifold degree of the associate line bundle. As it was mentioned in [3, Section $2.3]$, the result holds for any abstract geodesic flow. So here we apply this result for the geodesic flow given by the hyperbolic metric on $\mathcal{C}$ :

$$
\lambda_{1}=-\frac{2 \int_{\mathcal{C}} \alpha}{\chi(\mathcal{C})}=-\frac{2 \operatorname{deg} \overline{\mathbb{L}^{1,0}}}{\chi(\mathcal{C})} .
$$

This equality together with the previous proposition prove the second part of the theorem. The last statement of the theorem underlines the fact that any Teichmüller curve corresponding to a stratum of Abelian differentials admits a tautological bundle, which Lyapunov exponent is equal to 1.

This achieves the proof of the theorem.

\section{Acknowledgments}

The author is grateful to Quentin Gendron for helpful comments, to Martin Möller and Giovanni Forni for valuable remarks, to Anton Zorich for formulation of the problem, and to Carlos Matheus for careful reading the manuscript.

\section{References}

[1] I.I. Bouw and M. Möller, Teichmüller curves, triangle groups, and Lyapunov exponents, Ann. of Math. (2) 172(1) (2010), 139-185.

[2] P. Deligne, Un théorème de finitude pour la monodromie, in Discrete Groups in Geometry and Analysis, number 67 in Progr. Math., 1-19, Birkhäuser, Boston, MA (1987).

[3] A. Eskin, M. Kontsevich and A. Zorich, Lyapunov spectrum of square-tiled cyclic covers, J. Mod. Dyn. 5(2) (2011), 319-353.

[4] G. Forni, Deviation of ergodic averages for area-preserving flows on surfaces of higher genus, Ann. of Math. (2) 155(1) (2002), 1-103.

[5] G. Forni, C. Matheus and A. Zorich, Lyapunov spectrum of invariant subbundles of the Hodge bundle, arXiv:1112.0370v3.

[6] G. Forni, C. Matheus and A. Zorich, Zero Lyapunov exponents of the Hodge bundle, arXiv:1201.6075v3.

[7] J.H. Hubbard, Teichmüller theory and applications to geometry, topology, and dynamics. Vol. 1, Matrix Editions, Ithaca, NY (2006). 
[8] M. Kontsevich, Lyapunov exponents and Hodge theory, in The mathematical beauty of physics (Saclay, 1996), Vol. 24 of Adv. Ser. Math. Phys., 318-332, World Science Publ., River Edge, NJ (1997).

[9] M. Möller, Park City Lectures notes: Teichmüller curves, mainly from the viewpoint of algebraic geometry.

[10] M. Möller, Variations of Hodge structures of a Teichmüller curve, J. Amer. Math. Soc. 19(2) (2006), 327-344 (electronic).

[11] V.I. Oseledets, A multiplicative ergodic theorem, Trans. Moscow Math. Soc. 19 (1968), $197-231$.

[12] C. Peters, Arakelov-type inequalities for Hodge bundles, Prépublication de l'Institut Fourier 511 (2000), 1-11.

[13] C.A.M. Peters, A criterion for flatness of Hodge bundles over curves and geometric applications, Math. Ann. 268(1) (1984), 1-19.

[14] W. Schmid, Variation of Hodge structure: the singularities of the period mapping, Invent. Math. 22 (1973), 211-319.

[15] E. Viehweg and K. Zuo, Arakelov inequalities and the uniformization of certain rigid Shimura varieties, J. Different. Geom. 77(2) (2007), 291-352.

[16] A. Wilkinson, Conservative partially hyperbolic dynamics, in Proceedings of the International Congress of Mathematicians. Vol III, 1816-1836, Hindustan Book Agency, New Delhi (2010).

[17] A. Wright, Schwarz triangle mappings and Teichmüller curves: the Veech-Ward-Bouw-Möller curves, arXiv:1203.2685v2.

IRMAR, Université de Rennes 1, Campus de Beaulieu, 35042 Rennes, France

E-mail address: elise.goujard@univ-rennes1.fr 